\title{
Reflections on quality of care for persons with dementia: moving toward an integrated, comprehensive approach
}

With current projections indicating that the number of persons with dementia will continue to rise in the near future (Alzheimer Disease International, 2018), dementia is now recognized as a public health priority worldwide. Despite the great efforts and budgets invested; as of yet, no cure or highly effective treatments for dementia are available. Thus, the development of care practices to improve the wellbeing and quality of life of persons with dementia and their caregivers have become primary aims in the management of the condition (Ortega et al., 2018).

Caring for a person with dementia is a complex task, which requires paying attention to a variety of physical, psychological, emotional, social, and informational needs of the person him/herself, as well as those of the formal and informal caregivers. The papers published in this issue of International Psychogeriatrics reflect this complexity, and provide us with an opportunity to contemplate and discuss the need for an integrative care approach to maintain quality of life and wellbeing in persons with dementia.

\section{Overview of the papers in this issue}

For many years, reducing the deleterious effects associated with providing care to a relative has been one of the most important research and applied goals in the area of dementia care (Wennberg et al., 2015). Two of the papers in this issue study the effects of psychological factors that deal with the stress associated with caregiving. Ruisoto and colleagues (2018) examined associations between optimism and quality of life among 130 informal caregivers of persons with dementia. Based on a conceptualization of optimism as an integrated and multidimensional approach of generalized expectancies of control (p. 2), their study showed that optimism is a significant predictor of caregivers' quality of life beyond other covariates. Relying on a secondary analysis of data collected during a random control trial including 148 primary caregivers of persons with dementia, van der Lee and colleagues (2018) also explored factors associated with reduced caregiver burden. Caregivers with an improved sense of competence - defined as the subjective ability to cope with the responsibilities and duties of the caregiving role, and caring for a person with less behavioral problems - were found to have better chances of reducing their level of burden over time.

Together, these two studies suggest that caregivers with positive personality traits and higher levels of self-efficacy might be less vulnerable to the negative impact of caregiving. This provides support for developing interventions aimed at enhancing positive factors, rather than reducing negative ones, to improve caregivers' wellbeing, thereby allowing them to provide the best possible care for their loved ones.

El-Saifi and colleagues' (2018) study, which also focuses on informal caregivers, examines one of the most complicated, yet largely understudied, responsibilities of family caregivers of persons with dementia - securing appropriate medication adherence (George and Steffen, 2017). While previous studies have examined caregivers' characteristics, associated with their own medication adherence (Wang et al., 2015), to the best of my knowledge, this is the first study to assess caregivers' factors associated with care-receivers' medication adherence. Using Bayesian regression and based on caregivers' perspectives, these authors found a myriad of interrelated factors associated with medication adherence. Identifying different profiles of medication adherence is of great importance, as it might help recognize vulnerable dyads and provide additional support to caregivers in fulfilling this complex caregiving responsibility, while securing care-receivers' physical wellbeing.

The last two papers in this issue concentrate on other populations rather than informal caregivers. Shinan-Altman and colleagues (2018) used a qualitative methodology to explore the motivations of young adults to participate in a program aimed at training them as formal caregivers of older persons. While not specifically centered on the topic of providing care for persons with dementia, this study provides important insight regarding the intricate and sensitive task of appropriately motivating young persons to work with older adults.

Finally, Hendriks and colleagues (2018) described the challenges and impact of an interactive art program - called Unforgettable - for persons with dementia and their caregivers, implemented in 12 museums across the Netherlands. Analysis 
of semi-structured interviews conducted with 23 stakeholders involved in different implementation stages of the intervention, demonstrated the feasibility of the program, while underscoring the importance of following a clear plan. The results of structured interviews conducted with 176 persons working in the museums, but not directly involved in the intervention, showed improvement in their attitudes toward persons with dementia. This is an important finding, as it shows that implementing interventions outside a care environment benefits not only those directly involved in the program, but also the general public.

\section{Reflections on improving the quality of life of persons with dementia}

Reading this group of publications, we can learn about the unique contribution of each of the studies, but also about their commonalities and shared message. While assessing different targets (formal and informal caregivers, as well as laypersons), and using different methodologies (qualitative and quantitative), the underlying aspiration of these publications is to ensure and improve the quality of life of persons with dementia. This is indeed the major vision of recent global initiatives aimed at facing the challenge of dementia in future years (World Health Organization, 2019). Meeting this goal, however, requires an integrated, multifaceted approach, centering not only on the person with dementia, but also on formal and informal caregivers, as well as on the general population (Chertkow, 2018; World Health Organization, 2019).

While the relationships linking persons with dementia, family members and professionals' quality of life are well established in the research literature (Jing et al., 2016; Landeiro et al., 2018), their translation into an integrative model of care is still awaiting. The importance of developing a personcentered approach for the care of individuals with dementia (Manthorpe and Samsi, 2016), and the call of global initiatives to address the needs of all dementia stakeholders, including laypersons (Chertkow, 2018), are the first steps in this direction.

\section{Perla Werner}

Department of Community Mental Health, University of Haifa, Haifa, Israel

E-mail: Israel werner@research.haifa.ac.il

\section{References}

Alzheimer's Disease International (ADI) (2018). Available at: https://www.alz.co.uk/sites/default/files/pdfs/nationalplans-examples-2017.pdf; last accessed 30 January 2019.
Chertkow, H. (2018). An action plan to face the challenge of dementia: international statement on dementia from IAP for health. The fournal of Prevention of Alzheimer's Disease, 3, 208-213.

El-Saifi, N., Moyle, W., Jones, C. and Alston-Knox, C. (2018). Determinants of medication adherence in older people with dementia from the caregivers' perspective. International Psychogeriatrics, 31, 331-339.

George, N. R. and Steffen, A. M. (2017). Predicting perceived medication-related hassles in dementia family caregivers. Dementia, 16, 797-810.

Hendriks, I., Meilamd, F. J. M., Gerrisen, D. L. and Droes, R. M. (2018). Implementation and impact of unforgettable: an interactive art program for people with dementia and their caregivers. International Psychogeriatrics, 31, 351-362.

Jing, W., Willois, R. and Feng, Z. (2016). Factors influencing quality of life of elderly people with dementia and care implications: a systematic review. Archives of Gerontology and Geriatrics, 66, 23-41.

Landeiro, F. et al. (2018). Measuring quality of life of people with predementia and dementia and their caregivers: a systematic review protocol. BMF Open, 8, 8e019082, doi:10.1136/bmjopen-2017-019082.

Manthorpe, J. and Samsi, K. (2016). Person-centered dementia care: current perspectives. Clinical Interventions in Aging, 11, 1733-1740.

Ortega, V., Mukadam, N., Sommerlad, A. and Livingston, G. (2018). The Lancet commission on dementia prevention, intervention, and care: a call for action. Irish fournal of Psychological Medicine, doi:/10.1017/ipm.20184.

Ruisoto, P., Contador, I., Fernandez-Calvo, B., Palenzuela, D. and Ramos, F. (2018). Exploring the association between optimism and quality of life among informal caregivers of persons with dementia. International Psychogeriatrics, 31, 309-315.

Shinan-Altman, S., Riabzev, A. and Ayalon, L. (2018). Mixed motivations to provide formal care to older adults: lessons from a training program. International Psychogeriatrics, 31, 341-349.

van der Lee, J., Bakker, T. J. E. and Droes, R. M. (2018). Recovery from burden: informal caregiver profiles that predict treatment success. International Psychogeriatrics, 31, 317-329.

Wang, X., Robinson, K. M. and Harding, H. K. (2015). The impact of caregiving on caregivers' medication adherence and appointment keeping. Western fournal of Nursing Research, 37, 1548-1562.

Wennberg, A. Dye, Ch., Streetman-Loy, B. and Pham, H. (2015). Alzheimer's patient familial caregivers: a review of burden and interventions. Health $\mathcal{E}$ Social Work, 40, e162-e169.

World Health Organization (2019). Global Action Plan on the Public Health Response to Dementia: 2017-2025. Geneva: World Health Organization. Available at: https://apps.who.int/iris/bitstream/handle/10665/ 259615/9789241513487-eng.pdf;jsessionid= B43BBDA31CAF7DEE9B0DDDF285F44000? sequence $=1$; last accessed 5 February 2019 . 